\title{
Finite genome length corrections for the mean fitness and gene probabilities in evolution models
}

\author{
Zara Kirakosyan ${ }^{1}$, David B. Saakian ${ }^{1,2,3}$ * and Chin-Kun $\mathrm{Hu}^{2,4}$ 团 \\ ${ }^{1}$ Yerevan Physics Institute, Alikhanian Brothers St. 2, Yerevan 375036, Armenia \\ ${ }^{2}$ Institute of Physics, Academia Sinica, Nankang, Taipei 11529, Taiwan \\ ${ }^{3}$ National Center for Theoretical Sciences:Physics Division, \\ National Taiwan University, Taipei 1061\%, Taiwan and \\ ${ }^{4}$ Center for Nonlinear and Complex Systems and Department of Physics, \\ Chung Yuan Christian University, Chungli 32023, Taiwan
}

(Dated: August 22, 2018)

\begin{abstract}
Using the Hamilton-Jacobi equation approach to study genomes of length $L$, we obtain $1 / L$ corrections for the steady state population distributions and mean fitness functions for horizontal gene transfer model, as well as for the diploid evolution model with general fitness landscapes. Our numerical solutions confirm the obtained analytic equations. Our method could be applied to the general case of nonlinear Markov models.
\end{abstract}

PACS numbers: 87.10.+e, 87.15.Aa, 87.23.Kg, 02.50.-r

\section{INTRODUCTION}

After the development of molecular biology, the concepts and methods in statistical physics have been widely used to study molecular models of biological evolution [113], especially in recent years [14-20, 22 25]. Analytic solutions for models with the infinite genome length may be obtained via several methods, including the maximum principle 12, 13, Trotter-Suzuki method in quantum statistical physics [14, 15, 17, 18], quantum field theory 15, 18, 19 and the Hamiltonian-Jacobi equation method [21 23, 25]. The infinite genome length limit has been solved fora more complicated (more nonlinear) evolution model: the horizontal gene transfer model [16, 20, 23, 25]. This model has been solved both for the haploid [16, 20], and the hyper-geometric diploid case [7 9, 23].

The study of finite-size corrections is important not only for lattice phase transition models, but also for population genetics [26, 27]. Here there are situations with few relevant genes for the evolution problem. Although the genome length may be large, e.g. about 10000 in the case of HIV, it is possible to consider only the variable part of the genome which has only $40-100$ bases 28]; in such a case, the finite-size effects cannot be overlooked. The study of finite-size corrections allows us to understand as to what extent the results for the infinite genome length can be used to represent those for the finite genome length.

In recent works [19, 22] the finite genome length corrections have been calculated for the haploid molecular evolution model [4]. In [25] we calculated the finite size correction for the recombination model with single-peak fitness. In the present paper we calculate the finite genome length corrections for a diploid model with symmetric

\footnotetext{
*Electronic address: saakian@phys.sinica.edu.tw
}

${ }^{\dagger}$ Electronic address: hu@phys.sinica.edu.tw landscape 23] as well as for a haploid model with a simple horizontal gene transfer (HGT) [16, 20] for a general symmetric fitness landscape. The method may be applied to rather general cases of nonlinear probabilistic models 29].

Actually, we are constructing a perturbation expansion for the eigen-value like variable for the nonlinear operators. In case of linear operators (like the quantum mechanics), to calculate the first order perturbation to the energy we need only the 0 -th order expressions of eigen-vectors to calculate the leading corrections to eigenvalues due to perturbations. In case of nonlinear system of equations it is impossible to use the methods of linear algebra. Nevertheless, we can succeed using a trick. To calculate the perturbation expansion of eigen-value like variables (mean fitness or the asymptotic expression), we use equations such as Eq.(13), where the contribution of correction terms of the distribution function disappear. Thus the perturbation of mean-fitness ("eigen-value" for a nonlinear problem ) can be calculated using only the zeroth-order expression of eigen-functions (bulk solutions for probability distribution).

The present paper is organized as follows. In Section II we derive the finite size corrections for a haploid model (Crow-Kimura model) with a new method. In Sections III and IV the new method is applied to the diploid evolution model and HGT model, respectively. In the Appendix we give finite size corrections for the diploid evolution model with single-peak fitness function.

\section{FINITE SIZE CORRECTIONS FOR THE CROW-KIMURA MODEL}

Let us first introduce a new method to calculate finitesize corrections for the Crow-Kimura model with haploid genotypes [4], which is easier to study. The Crow-Kimura model is slightly easier to solve than the Eigen model [1, 2]. 
In the Crow-Kimura model [4], any genotype configuration $S_{i} \equiv\left(s_{1}^{i}, \ldots, s_{L}^{i}\right)$ is specified by a sequence of $L$ two-valued spins $S_{k}^{i}= \pm 1$ for $L \geq k \geq 1$ and $M-1 \geq i \geq 0$ such that the value +1 represents purines $(\mathrm{A}, \mathrm{G})$, and the value -1 represents pyrimidines $(\mathrm{T}, \mathrm{C})$, where $M=2^{L}$ is the total number of different sequences. The difference between two configuration $S_{i}$ and $S_{j}$ is described by the Hamming distance $d_{i j}=\left(L-\sum_{k} s_{k}^{i} s_{k}^{j}\right) / 2$. In other words, the Hamming distance is the number of different spins between configurations $S_{i}$ and $S_{j}$.

The relative frequency $x_{i}$ of a given configuration $S_{i}$ satisfies following equation:

$$
\frac{d x_{i}}{d t}=x_{i}\left(r_{i}-\sum_{j=0}^{M-1} r_{j} x_{j}\right)+\sum_{j=0}^{M-1} \mu_{i j} x_{j}
$$

here $r_{i}$ is the replication rate, i.e. the fitness of an organism with a given genotype and in Crow-Kimura's model it is specified like a function of the genotype: $r_{i}=L f\left(S_{i}\right)$. In other words fitness is the average number of offspring of individual with genotype sequence $S_{i}$ per unit period of time and it is very meaningful quantity in evolution theory. $\mu_{i j}$ is the mutation rate to move from sequence $S_{i}$ to sequence $S_{j}$ per unit period of time. It is important that in Crow-Kimura's model [4] only single base mutations are allowed, i. e. $\mu_{i j}=\mu$ for $d_{i j}=1, \mu_{i j}=0$ for $d_{i j} \neq 1$ and $i \neq j$, and $\mu_{i j}=-L \mu$ for $i=j$; the last condition ensures that the time evolution of $x_{i}$ does not change the normalization condition $\sum_{j=0}^{M-1} x_{j}=1$. In the following, we take $\mu=1$.

The nonlinear system of Eq. (1) can be mapped into following linear system of equations,

$$
\frac{d p_{i}}{d t}=p_{i} r_{i}+\sum_{j=0}^{M-1} \mu_{i j} p_{j}
$$

where $p_{i}$ are related to $x_{i}$ of Eq. (11) via [30, 31],

$$
x_{i}=\frac{p_{i}}{\sum_{j} p_{j}} \text {. }
$$

For the symmetric fitness landscape, where $r_{i}$ are the same for all the sequences with the same Hamming distance from the same reference sequence $S_{0} \equiv(1,1, \ldots, 1)$, it is more convenient to work with classes, i.e. we classify configurations $S_{i}$ into the classes according to the value $m_{l} \equiv m_{i}$, where $m_{i}$ is so-called "magnetization" of the configuration $S_{i}$ and is defined as $m_{i}=\sum_{k=1}^{L} s_{k}^{i} / L,-1 \leq$ $m^{i} \leq 1$.

Defining the magnetization $m_{l}$ for the configurations at the $l$-th class as $m_{l}=1-2 l / L$ and fitness $r_{i} \equiv L f\left(m_{l}\right)$, we rewrite Eq.(2) for the probability $p_{l}$ for a typical configuration in the $l$-class as

$$
\frac{d p_{l}}{d t}=p_{l}\left(L f\left(m_{l}\right)-L\right)+l p_{l-1}+(L-l) p_{l+1} .
$$

We define the $l$-th Hamming class as the group of all sequences at the Hamming distance $l$ from the reference sequences. Having the number of configurations at the $l$-th class $L_{l}=\frac{L !}{l !(L-l) !}$ for $P_{l}=L_{l} p_{l}$ we have

$$
\begin{aligned}
\frac{d P_{l}}{d t} & =P_{l}\left(L f\left(m_{l}\right)-L\right)+(L-l+1) P_{l-1} \\
& +(l+1) P_{l+1} .
\end{aligned}
$$

We are interested in calculating the mean fitness $R \equiv$ $\sum_{l} P_{l} f\left(m_{l}\right)$ and surplus $s \equiv \sum_{l} P_{l} m_{l}$ in the steady state. In [22], one of us proposed that in the steady state $\ln P_{l}$ and the mean fitness $R$ can be written as

$$
\begin{aligned}
\ln P_{l} & =L U(m, t)+O(1), \\
R & =L k+O(1),
\end{aligned}
$$

where $m=1-2 l / L$. Then, high order corrections for $R$, and for $\ln P_{l}$ have been derived using the linear algebra methods and the equations for $P_{l} / \sqrt{L_{l}}$. In this section we derive these corrections with an alternative method that can be applied to the strong nonlinear situations.

Using the ansatz $P_{l}=\exp [L U(m, t)]$ and a formula $P_{l \pm 1} \approx P_{l} e^{-\left( \pm 2 U_{m}^{\prime}\right)}$ where $U^{\prime} \equiv \frac{\partial U(m, t)}{\partial m}$, one can transform Eq. (5) into the Hamilton-Jacobi equation,

$$
\begin{aligned}
\frac{\partial U}{\partial t} & =H\left(m, U^{\prime}\right) \\
H(m, y) & \equiv f(m)-1+\frac{1+m}{2} e^{2 y}+\frac{1-m}{2} e^{-2 y}
\end{aligned}
$$

where $y \equiv U^{\prime}$ is a dummy variable. We assume an asymptotic

$$
U(m, t)=k t+u(m),
$$

and get an ordinary differential equation

$$
k=f(m)-1+\frac{1+m}{2} e^{2 u^{\prime}}+\frac{1-m}{2} e^{-2 u^{\prime}}
$$

$2 u^{\prime}=\ln \frac{k-1+f(m) \pm \sqrt{(k-1+f(m))^{2}-1+m^{2}}}{1+m},(9)$

which corresponds to Eq. (23) in Ref. 22]. We take the "+" solution for $-1 \leq m<m_{0}$ and " - " solution for $m>m_{0}$.

In Eq. (9), $k$ is a function $u^{\prime}$. The value of $u^{\prime}$, which gives the maximum value of $k$ is defined by equation

$$
u_{0}^{\prime}(m)=\frac{1}{4} \ln \frac{1-m}{1+m} .
$$

Thus the maximum value of $k$ can be obtained from

$$
\begin{aligned}
k & =\max [V(m)]_{-1 \leq m \leq 1} \\
V(m) & \equiv H\left(m, u_{0}(m)\right)=f(m)-1+\sqrt{1-m^{2}}
\end{aligned}
$$

where the maximum of the first equation is at the point $m_{0}$.

To calculate higher order corrections, we write $\ln P_{l}$ as

$$
\ln P_{l}=L\left(k+\frac{k_{1}}{L}\right) t+L u(m)+u_{1}(m)+O(1) .
$$


Equations (5) and (12) imply that 22]:

$$
\begin{aligned}
& k+\frac{k_{1}}{L}=f(m)-1+\frac{1+m}{2} e^{2 u^{\prime}}+\frac{1-m}{2} e^{-2 u^{\prime}} \\
+ & \frac{1}{L}\left(e^{2 u^{\prime}}+e^{-2 u^{\prime}}\right)+\frac{2 u^{\prime \prime}}{L}\left(\frac{1+m}{2} e^{2 u^{\prime}}+\frac{1-m}{2} e^{-2 u^{\prime}}\right) \\
+ & \frac{2 u_{1}^{\prime}}{L}\left[\frac{1+m}{2} e^{2 u^{\prime}}-\frac{1-m}{2} e^{-2 u^{\prime}}\right] .
\end{aligned}
$$

In Eq. (13), $k$ is the bulk expression of the mean fitness and $k_{1} / L$ is the first order correction to it. Having the value of $k_{1}$, we can calculate $u_{1}^{\prime}$. $k_{1}$ can be defined from Eq.(13) at the point $m=m_{0}$, where the coefficient of $u_{1}^{\prime}$ becomes zero. We have an equation

$$
e^{2 u^{\prime}\left(m_{0}\right)}=\sqrt{\frac{1-m_{0}}{1+m_{0}}} .
$$

Equation (13) then implies that at $m_{0}$

$$
\begin{aligned}
k_{1} & =e^{2 u^{\prime}}+e^{-2 u^{\prime}}+2 u^{\prime \prime}\left(\frac{1+m}{2} e^{2 u^{\prime}}+\frac{1-m}{2} e^{-2 u^{\prime}}\right) \\
& =2 \frac{1}{\sqrt{1-m_{0}^{2}}}+2 u^{\prime \prime}\left(m_{0}\right) \sqrt{1-m_{0}^{2}} .
\end{aligned}
$$

Let us define $u^{\prime \prime}\left(m_{0}\right)$. Equation (9) can be written as $k=H\left(m, p=u^{\prime}\right)$. Expanding Eq. (9) near $m=m_{0}$ with respect to the first and the second arguments of $H\left(m, p=u^{\prime}\right)$ up to the second order, we derive

$$
\begin{aligned}
0 & \approx V^{\prime \prime}\left(m_{0}\right) \frac{\left(m-m_{0}\right)^{2}}{2} \\
& +H_{p p}^{\prime \prime}\left(m_{0}, u_{0}\left(m_{0}\right)\right) \frac{\left(u^{\prime}(m)-u_{0}^{\prime}(m)\right)^{2}}{2} .
\end{aligned}
$$

Note that there is no $\left(m-m_{0}\right)\left(u^{\prime}-u_{0}^{\prime}\right)$ term in the last equation. We can verify this directly: $H_{p}^{\prime}\left(m, p_{0}\right)=0$.

From Eqs. (7), (11) and (10), we derive

$$
\begin{gathered}
H_{p p}^{\prime \prime}=4 \sqrt{1-m_{0}^{2}}, \\
V^{\prime \prime}\left(m_{0}\right)=f^{\prime \prime}\left(m_{0}\right)-\frac{1}{\left(1-m_{0}^{2}\right)^{3 / 2}}, \\
u_{0}^{\prime}(m) \approx-\left(m-m_{0}\right) \frac{1}{2\left(1-m_{0}^{2}\right)}+u_{0}^{\prime}\left(m_{0}\right) .
\end{gathered}
$$

With these expressions in Eq.(16), we derive

$f^{\prime \prime}\left(m_{0}\right)=\frac{1}{\left(1-m_{0}^{2}\right)^{3 / 2}}-\sqrt{1-m_{0}^{2}}\left(2 u^{\prime \prime}\left(m_{0}\right)+\frac{1}{1-m_{0}^{2}}\right)^{2}$.

Therefore

$$
\begin{aligned}
2 u^{\prime \prime}\left(m_{0}\right) & =-\frac{1}{\left(1-m_{0}^{2}\right)} \\
& -\frac{1}{\left(1-m_{0}^{2}\right)^{1 / 4}} \sqrt{f^{\prime \prime}-\frac{1}{\left(1-m_{0}^{2}\right)^{3 / 2}}} .
\end{aligned}
$$
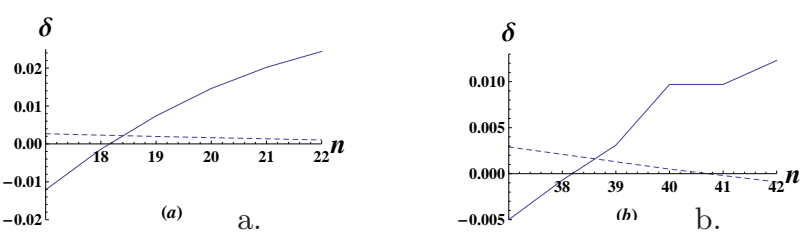

FIG. 1: Comparison of the 0 -th order and $1 / L$ accuracy expressions for the haploid model class probabilities with the numerics given by Eq.(4) for the fitness function $f(m)=\frac{1}{2} m^{2}$. $p_{n}$ are the results of numerics for the original model given by the system of equations. $p_{0 n} \equiv \exp (L u(m))$ is calculated using $O(L)$ terms in expression of $\ln p_{n}$, Eq.(12), while $p_{1 n} \equiv \exp \left(L u(m)+u_{1}(m)\right)$ corresponds to the $O(1)$ accuracy in expression of $\ln p_{n}$, Eq. (12). The smooth line corresponds to $\delta=\ln p_{0 n} / p_{n}$. For Fig 1.a $L=80$ and the dashed line corresponds to $\delta=\ln p_{1 n} / p_{n}$. For Fig 1.b $L=160$ and the dashed line corresponds to $\delta=10 \ln p_{1 n} / p_{n}$.

Equations (10) and (14) imply that $u^{\prime}\left(m_{0}\right)=u_{0}^{\prime}\left(m_{0}\right)$. Thus eventually, we can use Eq. (15) to obtain [19], 22]

$$
\begin{aligned}
& k_{1}=\frac{1}{\sqrt{1-m_{0}^{2}}} \\
& -\sqrt{\frac{1}{\left(1-m_{0}^{2}\right)^{3 / 2}}-f^{\prime \prime}\left(m_{0}\right)}\left(1-m_{0}^{2}\right)^{1 / 4} \\
= & \frac{1}{\sqrt{1-m_{0}^{2}}}\left[1-\sqrt{1-\left(1-m_{0}^{2}\right)^{3 / 2} f^{\prime \prime}\left(m_{0}\right)}\right] .
\end{aligned}
$$

Using the expression for $k_{1}$, we can calculate the $u_{1}^{\prime}$ from Eq. (13):

$$
\begin{aligned}
& 2 u_{1}^{\prime}\left[\frac{1+m}{2} e^{2 u^{\prime}}-\frac{1-m}{2} e^{-2 u^{\prime}}\right] \\
= & k_{1}-\left(e^{2 u^{\prime}}+e^{-2 u^{\prime}}\right) \\
- & 2 u^{\prime \prime}\left(\frac{1+m}{2} e^{2 u^{\prime}}+\frac{1-m}{2} e^{-2 u^{\prime}}\right) .
\end{aligned}
$$

Therefore, having $u_{1}$, Eq.(19), we can calculate the $1 / L$ accuracy expression for the haploid class probabilities $P_{l}$ (we define them as $\hat{p_{1 l}} \equiv\left(L u(m)+u_{1}(m)\right)$ ), also we can calculate the probabilities $p_{0 l} \equiv e^{L u(m)}$, Eq. (12). The comparison of this computations with numerical results are shown in the Fig. 1 for the case $L=80,160$.

On the other hand, we can calculate $k_{1}$ directly as $\sum_{l} f\left(m_{l}\right) P_{l}-k$,

$$
k_{1}=\frac{1}{\left|u^{\prime \prime}(s)\right|}\left(f^{\prime}(s) u_{1}^{\prime}(s)+\frac{f^{\prime \prime}(s)}{2}\right) .
$$

The bulk surplus term is calculated using the equation 12.

$$
f(s)=R .
$$

From the definition of $s$, the correction to the surplus can be written as $\delta s=\frac{u_{1}^{\prime}(s)}{L\left|u^{\prime \prime}(s)\right|}$. Comparing with Eq. (20), 
we get $\delta s=\left(k_{1}-\frac{f^{\prime \prime}(s)}{2\left|u^{\prime \prime}(s)\right|}\right) /\left(L f^{\prime}(s)\right)$. Using the formula

$$
u^{\prime \prime}(s)=-\frac{f^{\prime}(s)}{2 s},
$$

we derive, assuming $f^{\prime}(s)>0$ :

$$
\delta s=\left(k_{1}-\frac{s f^{\prime \prime}(s)}{f^{\prime}(s)}\right) \frac{1}{L f^{\prime}(s)} .
$$

One can define $u_{1}(m)$ integrating the expression of $u_{1}^{\prime}(m)$ from Eq. (19). Thus we have derived the results of [22] by an alternative method.

\section{FIRST ORDER CORRECTIONS FOR THE DIPLOID CASE}

The diploid model has been defined by Eq. (1) in 23]. There we derived the analytic solution for the diploid many allele biological evolution models $7[-9]$ with general fitness landscapes to the first order using the HamiltonJacobi equation approach. In the present paper we will find $1 / L$ accuracy for mean fitness and genome probabilities of that diploid model.

In WBS parallel diploid model [8], gene probabilities $p_{i}$ evolve as

$$
\frac{d p_{i}}{d t}=p_{i}\left[\sum_{j=1}^{M} A_{i j} p_{j}-\sum_{k=1}^{M} \sum_{j=1}^{M} A_{j k} p_{j} p_{k}\right]+\sum_{j=1}^{M} m_{i j} p_{j} .
$$

Here $m_{i j}$ is a mutation matrix, defined in Section II. We have a balance condition $\sum_{i=1}^{M} p_{i}=1 . A_{i j}$ is the fitness of the genotype $\left(S_{i}, S_{j}\right)$, and $\sum_{j} A_{i j} p_{j}$ is the marginal fitness for the sequence $S_{i}$.

We assume that the fitness of the configuration $S_{i}$ is a smooth function of the Hamming distance between $S_{i}$ and the reference configuration $S_{1}$. In such case, it is convenient to work with the overlap $m=\left(1-2 d_{i 1} / L\right)$ instead of the Hamming distance $d_{i 1}$. Consider the following choice of the matrix $\hat{A}$ :

$$
\begin{aligned}
& A_{i i}=f(m, m), \\
& A_{i j}=f\left(m_{1}, m_{2}\right),
\end{aligned}
$$

where $m_{1}=1-2 d_{i 1} / L, m_{2}=1-2 d_{j 1} / L$. In the hypergeometric model $L\left(1+m_{1}\right) / 2$ and $L\left(1+m_{2}\right) / 2$ are, respectively, the number of $A_{1}$ maternal and paternal alleles. $f\left(m_{1}, m_{2}\right)$ is a smooth analytical function. We are interested in finding the exact phase structure and the steady state, therefore we can consider only symmetric solutions of $p_{i}$.

Using the expression $P_{l} \sim \exp [R t+\ldots]$ in Eq. (13) of [23], we obtain coupled equations for $P_{l}$ that describe the diploid, one locus many allele parallel mutation-selection model [23, 32]:

$$
\begin{aligned}
R P_{l} & =L P_{l} F_{l}+(L-l+1) P_{l-1}+(l+1) P_{l+1} \\
& -L P_{l}\left(1+\sum_{k} F_{k} P_{k}\right) .
\end{aligned}
$$

Here we define the marginal fitness for the Hamming class $F_{l}=\sum_{n=0}^{L} f(1-2 l / L, 1-2 n / L) P_{n}$. In diploid case the fitness landscape is defined via a function $f\left(m_{1}, m_{2}\right)$ of two arguments, describing the dominance relations among alleles 7-9]. Eq.(21) coincides with the CrowKimura model where $F_{l}$ is the fitness function for the Hamming class 1 .

Assuming ansatz (6) and (8), we derive the bulk expression for steady state solution as

$$
k=f(m, s)-1+\frac{1+m}{2} e^{2 u^{\prime}(m)}+\frac{1-m}{2} e^{-2 u^{\prime}(m)},
$$

where $u(m)$ is defined via Eqs.(6),(8). The mean fitness per spin $k$ and surplus $s \equiv \sum_{l} P_{l}(1-2 l / L)$ are defined via a system [23]

$$
\begin{aligned}
k & =f(s, s), \\
f_{m}^{\prime}\left(m_{0}, s\right) & -\frac{m_{0}}{\sqrt{1-m_{0}^{2}}}=0 .
\end{aligned}
$$

To find an expression for $F_{l}$ with $1 / L$ accuracy, we calculate the following integral:

$$
F_{l}=\int f(m, x) e^{L u(x)+u_{1}(x)} d x,
$$

where $m=1-2 l / L$. Therefore we derive

$$
\begin{aligned}
F_{l}-f(m, s) & =\frac{f_{b}^{\prime}(m, s)\left[u_{1}^{\prime}(s)\right]}{L\left|u^{\prime \prime}(s)\right|}+\frac{f_{b b}^{\prime \prime}[m, s]}{2 L\left|u^{\prime \prime}(s)\right|} \\
& \equiv f_{b}^{\prime}(m, s) \frac{A}{L}+\frac{f_{b b}^{\prime \prime}[m, s]}{2 L\left|u^{\prime \prime}(s)\right|}
\end{aligned}
$$

where $A=\left[u_{1}^{\prime}(s)\right] /\left|u^{\prime \prime}(s)\right|, s$ is the surplus, $f_{b}^{\prime}(m, s)$ is the first derivative of the fitness function via the second argument, and $f_{b b}^{\prime \prime}(m, s)$ is the second derivative of the fitness function via the second argument.

For the mean fitness $R / L \equiv k+\frac{k_{1}}{L}$, from Eq. (29) we have

$$
\begin{aligned}
\frac{R}{L} & =\sum_{l} F_{l} P_{l}=\int f(m, s) e^{L u(m)+u_{1}(m)} d m \\
& +f_{b}^{\prime}(m, s) \frac{A}{L}+\frac{f_{b b}^{\prime \prime}[m, s]}{2\left|u^{\prime \prime}(s)\right|} .
\end{aligned}
$$

Expanding $f(m, s)$ via the first argument in the equation above, we obtain

$$
k_{1}=\left[f_{b}^{\prime}(s, s)+f_{a}^{\prime}(s, s)\right] \frac{A}{L}+\frac{\left[f_{b b}^{\prime \prime}[s, s]+f_{a a}^{\prime \prime}[s, s]\right.}{2 L\left|u^{\prime \prime}(s)\right|}
$$

with $f_{a}^{\prime}(m, s)$ being the first derivative of the fitness function via the first argument and $f_{a a}^{\prime}(m, s)$ being the second derivative of the fitness function via the first argument. On the other hand, we can get an expression for $k_{1}$ by adding the term $f_{b}^{\prime}(m, s) \frac{A}{L}+\frac{f_{b b}^{\prime \prime}[m, s]}{2\left|u^{\prime \prime}(s)\right|}$ to the right-hand 
side of Eq.119). Thus we obtain the following equation for the corrections:

$$
\begin{aligned}
& {\left[f_{b}^{\prime}(s, s)+f_{a}^{\prime}(s, s)\right] A+\frac{\left[f_{b b}^{\prime \prime}[s, s]+f_{a a}^{\prime \prime}[s, s]\right.}{2\left|u^{\prime \prime}(s)\right|} } \\
= & 2 u_{1}^{\prime}\left[\frac{1+m}{2} e^{2 u^{\prime}}-\frac{1-m}{2} e^{-2 u^{\prime}}\right] \\
+ & f_{b}^{\prime}(m, s) A+\frac{f_{b b}^{\prime \prime}[m, s]}{2\left|u^{\prime \prime}(s)\right|} \\
+ & 2 u^{\prime \prime}\left[\frac{1+m}{2} e^{2 u^{\prime}}+\frac{1-m}{2} e^{-2 u^{\prime}}\right]+2 \cosh \left(2 u^{\prime}\right) .
\end{aligned}
$$

The first line is derived via direct integration of the fitness function via the steady state distribution, the $u_{1}^{\prime}$ term in the second line is just the small $u_{1}^{\prime}$ correction of the right hand side of Eq.(26). The third line corresponds to Eq. (29). The last line corresponds to the correction terms of the haploid model.

To calculate $k_{1}$ we again consider the point where the coefficient of $u_{1}^{\prime}$ disappears in the last equation. We calculate $u^{\prime \prime}\left(m_{0}\right)$ as in the previous section. We have an equation for $A$

$$
\begin{aligned}
& {\left[f_{b}^{\prime}(s, s)+f_{a}^{\prime}(s, s)-f_{b}^{\prime}\left(m_{0}, s\right] A\right.} \\
= & \frac{\left.f_{b b}^{\prime \prime}\left(m_{0}, s\right)-f_{a a}^{\prime \prime}(s, s)-f_{b b}^{\prime \prime}(s, s)\right]}{2\left|u^{\prime \prime}(s)\right|} \\
+ & \frac{1}{\sqrt{1-m_{0}^{2}}}\left[1-\sqrt{1-f_{a a}^{\prime \prime}\left(m_{0}, s\right)\left(1-m_{0}^{2}\right)^{3 / 2}}\right] \\
+ & \frac{\left.f_{b b}^{\prime \prime}\left(m_{0}, s\right)-f_{a a}^{\prime \prime}(s, s)-f_{b b}^{\prime \prime}(s, s)\right]}{2\left|u^{\prime \prime}(s)\right|}
\end{aligned}
$$

where $m_{0}$ is the bulk magnetization. Then putting the value of $A$ in Eq. (33), we get an expression for $k_{1}$ :

$$
\begin{gathered}
k_{1}=\frac{f_{a a}^{\prime \prime}(s, s)+f_{b b}^{\prime \prime}(s, s)}{2\left|u^{\prime \prime}(s)\right|} \\
+\frac{f_{b}^{\prime}(s, s)+f_{a}^{\prime}(s, s)}{f_{b}^{\prime}(s, s)+f_{a}^{\prime}(s, s)-f_{b}^{\prime}\left(m_{0}, s\right)} \\
\times\left[\frac{f_{b b}^{\prime \prime}\left(m_{0}, s\right)-f_{a a}^{\prime \prime}(s, s)-f_{b b}^{\prime \prime}(s, s)}{2\left|u^{\prime \prime}(s)\right|}+k_{1 h}\right], \quad \\
k_{1 h}=\frac{1}{\sqrt{1-m_{0}^{2}}}\left[1-\sqrt{1-f_{a a}^{\prime \prime}\left(m_{0}, s\right)\left(1-m_{0}^{2}\right)^{3 / 2}}\right],
\end{gathered}
$$

where

$$
u^{\prime \prime}(s)=-\frac{f_{a}^{\prime}(s, s)}{2 s} .
$$

From Eq. 27) we calculate first order accuracy expression for the diploid mean fitness $k_{\text {theor }} \equiv k_{0}$ and then from Eq.(34) we compute the $1 / L$ accuracy expression for mean fitness $k=k_{0}+k_{1} / L$. Having all these expressions, we compare our analytical results with the direct numerics for different values of $L$ and different values of $a$ in Table I.

\begin{tabular}{|c|c|c|c|c|c|c|}
\hline$L$ & 100 & 100 & 100 & 150 & 150 & 150 \\
\hline$a$ & 4.5 & 5.5 & 6 & 4.5 & 5.5 & 6 \\
\hline$k_{n}$ & 3.18506 & 4.15619 & 4.64491 & 3.18455 & 4.15574 & 4.64449 \\
\hline$\delta_{0}^{\prime}$ & 0.00152 & 0.00132 & 0.00124 & 0.001014 & 0.000883 & 0.000829 \\
\hline$\delta_{1}^{\prime}$ & 0.00001 & 0.00001 & 0.00001 & 0.000005 & 0.000005 & 0.000005 \\
\hline
\end{tabular}

TABLE I: Comparison of 0-th order accuracy expression $\delta_{0}^{\prime} \equiv$ $k_{n}-k_{0}$ and the $1 / \mathrm{L}$ accuracy expression $\delta_{1}^{\prime} \equiv k_{n}-k$ results for the mean fitness of diploid model, for the fitness function $f_{0}\left(m_{1}, m_{2}\right)=\frac{a}{2}\left(m_{1}^{2}+m_{2}^{2}\right)+b m_{1} m_{2}$, for $b=0.5 . k_{n}$ is given by direct numerics of Eq.(4).
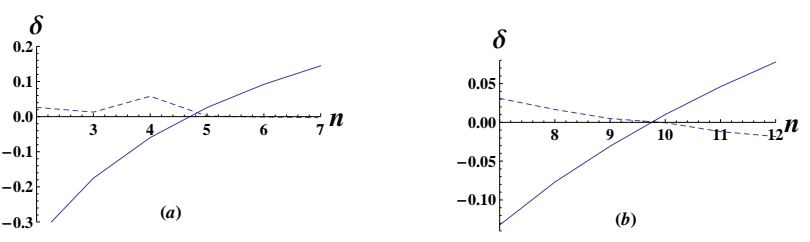

FIG. 2: Comparison of 0-th order accuracy expression and the $1 / \mathrm{L}$ accuracy expression for the $p_{n}$ in diploid model, for the fitness function $f_{0}\left(m_{1}, m_{2}\right)=\frac{a}{2}\left(m_{1}^{2}+m_{2}^{2}\right)+b m_{1} m_{2}$ and $a=4.5, b=0.5 . \quad p_{n}$ are the results of numerics for the of the original model given by the system of equations. $p_{0 n}=$ $\exp (L u(m))$ is calculated using $O(L)$ terms in expression of $\ln p_{n}$, Eq.(12), while $p_{1 n}=\exp \left(L u(m)+u_{1}(m)\right)$ corresponds to the $O(1)$ accuracy in expression of $\ln p_{n}$, Eq. (12). $\delta=$ $\ln p_{0 n} / p_{n}$, the smooth line. For Fig 2.a $L=50 \delta=\ln p_{1 n} / p_{n}$ is the dashed line. For Fig 2.b $L=100$, and $\delta=10 \ln p_{2 n} / p_{n}$ is the dashed line.

Having $u_{1}$ by Eq.(32), we calculate the $1 / L$ accuracy expression for the diploid class probabilities $p_{2 n}=$ $\exp \left(L u(m)+u_{1}(m)\right)$. In Fig. 2 we give the comparison of our analytical results with the direct numerics.

\section{HORIZONTAL GENE TRANSFER MODEL}

The finite genome length effects are very important for the case of horizontal gene transfer(HGT). While the genome length could be large (about 10000 in case of $\mathrm{HIV}$ ), it is possible to consider only the variable part of genome, $L=40-100[28]$ ). We consider the model [16, 20] describing a simple horizontal gene transfer. The model has been solved 20 in the large genome limit in 20] (mean fitness) and 23] (steady state distribution).

In this model, besides the mutation process, one spin in the genome is replaced with the spin at the same position from the sequence pool. 
We consider the following system of equations [16, 20]:

$$
\begin{aligned}
& \frac{d P_{l}}{d t}=P_{l} r_{l}+\left[(L-l+1) P_{l-1}+(l+1) P_{l+1}\right]- \\
& P_{l}\left(1+\frac{1}{L} \sum_{k} r_{k} P_{k}\right)- \\
& c P_{l}+c\left(\frac{\bar{l}}{L} \frac{l}{L}+\frac{L-l}{L} \frac{L-\bar{l}}{L}\right) P_{l}+ \\
& \left.c\left(1-\frac{l-1}{L}\right) \frac{\bar{l}-1}{L} P_{l-1}+c \frac{l+1}{L}\left(1-\frac{\bar{l}+1}{L}\right) P_{l+1}\right](36)
\end{aligned}
$$

where $\hat{l}=\sum_{l} P_{l} l=(1-s) L / 2$. The first and second lines correspond to the Crow-Kimura model [4], [12]. The third line describes the HGT process with the change of the Hamming class. We cut plus spin with the probability $(1-l / L)$ and add plus spin from the sequence pool with the probability $(1-\bar{l} / L)$, then cut minus spin with probability $l / L$ and add minus spin from the sequence pool with the probability $\bar{l} / L$. The last line corresponds to the process with the change of Hamming class. We cut the plus spin with the probability $(l-1) / L$ and add minus spin from sequence pool with the probability $1-(\bar{l}-1) / L$, then cut the minus spin with the probability $(l+1) / L$ and add plus spin from sequence pool with the plus spin with the probability $1-(\bar{l}+1) / L$.

Considering the ansatz

$$
P_{l}=\exp \left[L\left(k+\frac{k_{1}}{L}\right) t+L u(m)+u_{1}(m)\right],
$$

we get the following equation for the bulk terms

$$
\begin{aligned}
& k=H\left(m, u^{\prime}\right) \\
& H(m, p) \equiv f(m)+\frac{1+m}{2} e^{2 p}\left[1+\frac{c(1-s)}{2}\right] \\
& +\frac{1-m}{2} e^{-2 p}\left[1+c \frac{1+s}{2}\right]+\frac{c m s}{2}-\frac{c}{2}-1,
\end{aligned}
$$

where $r_{l}=L f(m)$ and $p$ is a dummy variable. The minimum of $H\left(m, u^{\prime}\right)$ is at $u^{\prime}=u_{0}(m)$, where

$$
e^{4 u_{0}(m)}=\frac{(1-m)\left(1+c \frac{1+s}{2}\right)}{(1+m)\left(1+c \frac{1-s}{2}\right)}
$$

gives a potential $V(m, s) \equiv \min \left[H\left(m, u^{\prime}\right)\right]_{u^{\prime}}$ :

$$
\begin{aligned}
& V(m, s)=f(m)+\sqrt{\left(1-m^{2}\right) C}+\frac{c m s}{2}-\frac{c}{2}-1, \\
& C=\left[\left(1+\frac{c}{2}\right)^{2}-\frac{c^{2} s^{2}}{4}\right] .
\end{aligned}
$$

We define $m_{0}, s$ from the conditions

$$
V^{\prime}\left(m_{0}, s\right)=0, \quad f(s)=V\left(m_{0}, s\right),
$$

where the derivative is with respect to the first argument. Let us calculate the $u^{\prime \prime}\left(m_{0}\right)$. Near $m_{0}$ we have an expansion

$$
\begin{aligned}
0 & \approx V^{\prime \prime}\left(m_{0}, s\right) \frac{\left(m-m_{0}\right)^{2}}{2} \\
& +H_{p p}^{\prime \prime}\left(m_{0}, u_{0}^{\prime}\right) \frac{\left(u^{\prime}(m)-u_{0}(m)\right)^{2}}{2}
\end{aligned}
$$

where $V^{\prime \prime}$ is the second derivative of $V$ with respect to the first argument, $H_{p p}^{\prime}$ is the second partial derivative of $H^{\prime}$ with respect to the second argument of the function $H(m, p)$. Dividing Eq. (41) by $\left(m-m_{0}\right)^{2}$, we derive the equation

$$
f^{\prime \prime}\left(m_{0}\right)=\frac{\sqrt{C}}{\left(1-m_{0}^{2}\right)^{3 / 2}}-\sqrt{\left(1-m^{2}\right) C}\left(u^{\prime \prime}-u_{0}^{\prime \prime}\right)^{2}
$$

or

$$
2 u^{\prime \prime}\left(m_{0}\right)=-\frac{1}{\left(1-m_{0}^{2}\right)}-\frac{\sqrt{-f^{\prime \prime}+\frac{\sqrt{C}}{\left(1-m_{0}^{2}\right)^{3 / 2}}}}{\left[\left(1-m_{0}^{2}\right) C\right]^{1 / 4}} .
$$

From Eq.(36) we have:

$$
\begin{aligned}
k_{1}= & \frac{f^{\prime}(s) u_{1}^{\prime}(s)}{\left|u^{\prime \prime}\right|}+\frac{f^{\prime \prime}(s)}{2 u^{\prime \prime}(s)} \\
= & 2 u_{1}^{\prime}\left[e^{2 u^{\prime}} \frac{1+m}{2}\left(1+c \frac{1-s}{2}\right)\right. \\
& \left.-\frac{1-m}{2}\left(1+c \frac{1+s}{2}\right) e^{-2 u^{\prime}}\right] \\
+ & {\left[e^{2 u^{\prime}}\left(1+c \frac{1-s}{2}\right)+\left(1+c \frac{1+s}{2}\right) e^{-2 u^{\prime}}\right] } \\
+ & 2 u^{\prime \prime}\left[e^{2 u^{\prime}} \frac{1+m}{2}\left(1+c \frac{1-s}{2}\right)\right. \\
& \left.+\frac{1-m}{2}\left(1+c \frac{1+s}{2}\right) e^{-2 u^{\prime}}\right] \\
+ & c u_{1}^{\prime}(s) \frac{m-e^{2 u^{\prime}} \frac{1+m}{2}+e^{-2 u^{\prime}} \frac{1-m}{2}}{2\left|u^{\prime \prime}\right|} .
\end{aligned}
$$

The first line is a direct integration of the fitness via steady state distribution, the second line corresponds to the correction of $F(m, p)$ via $\delta p \equiv u_{1}^{\prime}$, the third line corresponds to the corrections in the first line of Eq. (36). The last line corresponds to the $\delta s$ corrections from the $\hat{l}$ terms in Eq.(36).

To calculate $k_{1}$, we put the optimal value of $u^{\prime}$ and look at the point $m=m_{0}$. Then Eq.(44) is simplified:

$$
\begin{aligned}
& \frac{f^{\prime}(s) u_{1}^{\prime}(s)}{\left|u^{\prime \prime}\right|}+\frac{f^{\prime \prime}(s)}{2\left|u^{\prime \prime}(s)\right|} \\
= & \frac{2}{\sqrt{1-m_{0}^{2}}} \sqrt{C}+2 u^{\prime \prime}\left(m_{0}\right) \sqrt{1-m_{0}^{2}} \sqrt{C} \\
& +c u_{1}^{\prime}(s) \frac{m_{0}-\frac{c s \sqrt{1-m_{0}^{2}}}{2 \sqrt{C}}}{2\left|u^{\prime \prime}(s)\right|} \\
= & c u_{1}^{\prime}(s) \frac{m_{0}-\frac{c s \sqrt{1-m_{0}^{2}}}{2 \sqrt{C}}}{2\left|u^{\prime \prime}(s)\right|} \\
+ & \frac{\sqrt{C}}{\sqrt{1-m_{0}^{2}}}\left[1-\sqrt{1-\frac{f^{\prime \prime}\left(1-m_{0}^{2}\right)^{3 / 2}}{\sqrt{C}}}\right] .
\end{aligned}
$$

Deriving $u_{1}^{\prime}(s)$ from the last equation, we obtain

$$
k_{1}=\frac{f^{\prime}(s) u_{1}^{\prime}(s)}{\left|u^{\prime \prime}\right|}+\frac{f^{\prime \prime}(s)}{2\left|u^{\prime \prime}(s)\right|} .
$$


Thus

$$
\begin{gathered}
k_{1}=\frac{f^{\prime \prime}(s) s}{f^{\prime}(s)}+\frac{f^{\prime}(s)}{f^{\prime}(s)-c \frac{m_{0}-\frac{c s \sqrt{1-m_{0}^{2}}}{2 \sqrt{C}}}{2}} \times \\
{\left[\frac { \sqrt { C } } { \sqrt { 1 - m _ { 0 } ^ { 2 } } } \left[1-\sqrt{\left.\left.1-\frac{f^{\prime \prime}\left(m_{0}\right)\left(1-m_{0}^{2}\right)^{3 / 2}}{\sqrt{C}}\right]-\frac{f^{\prime \prime}(s)}{f^{\prime}(s)}\right]}\right.\right.}
\end{gathered}
$$

For the fitness function $f(m)=\mathrm{cm}^{2}$ with parameters $c=1$, we derive from the numerics for the mean fitness and order parameters $R / L=0.218142, m_{0}=$ $0.765984, s=0.467057$. The analytical formula Eq. 400) for the infinite genome limit gives $k=0.21842$, while the numerics for $L=100$ gives $R / L=0.21973$ and for $L=200, R / L=0.218946$. Thus we get for $L=100$, $R / L-k=0.00158, R / L-k-k_{1} / L=0.00002$ and for $L=200, R / L-k=0.000803, R / L-k-k 1 / L=0.000003$. We see that our analytical result by Eq. (47) for the mean fitness expression is well confirmed.

One can define $u_{1}(m)$ by integrating the expression of $u_{1}^{\prime}(m)$ from Eq. (45).

\section{DISCUSSION}

We calculated the finite genome size corrections to the mean fitness and steady state distribution for strongly nonlinear evolution models: horizontal gene transfer model and diploid parallel evolution model for general fitness function. The application of the Hamilton-Jacobi equation for the investigation of the master equation, especially the finite size corrections, is a rather popular method in case of linear master equation for chemistry or ecology [34, 35]. For the models, considered in our article, the standard methods of linear algebra and the methods of [34, 35] fail.

The key point of our method is that we investigate the point where the coefficient of the correction to the steady state distribution disappears. Then the correction to the fitness is calculated from the smoothness condition. Our method could be applied to other nonlinear probabilistic models as well.

Our formulas could be applied for relatively small genome lengths, for the single peak fitness landscape even for $L \sim 4$, see Appendix A. In population genetics usually few allele models are investigated with $L=2,3$. Fortunately, for the larger allele numbers we can apply our methods. Our formulas, while a bit involved, are less cumbersome than the formulas for several allele cases in population genetics.

DBS thanks DARPA Prophecy Program and Academia Sinica for financial support.
Appendix A. Finite $L$ corrections for a parallel diploid model with the single-peak fitness function

Consider the following diploid model studied in [23]:

$$
\begin{aligned}
\frac{d p_{i}}{d t} & =p_{i}\left[\sum_{j} A_{i j} p_{j}-\sum_{l=1}^{2^{L}} \sum_{j=1}^{2^{L}} A_{j l} p_{j} p_{l}\right] \\
& +\sum_{j} m_{i j} p_{j}
\end{aligned}
$$

with the fitness coefficients

$$
\begin{aligned}
& A_{11}=2 s ; A_{1 i}=A_{i 1}=2 s h, \text { for } i \neq 1 \\
& A_{i j}=0, \text { for } i>1, j>1 .
\end{aligned}
$$

Here $m_{i j}$ is the mutation matrix, $m_{i i}=-1, m_{i j}=1 / L$ for $d_{i j}=1$, and for other cases $m_{i j}=0$.

For the steady state we have [8, 23]

$$
\begin{aligned}
R p_{i} & =p_{i} \sum_{j} A_{i j} p_{j}+\sum_{j} m_{i j} p_{j} \\
R & =\sum_{l=1}^{2^{L}} \sum_{j=1}^{2^{N}} A_{j l} p_{j} p_{l} \\
& =2 s\left[(1-2 h) x^{2}+2 h x\right] .
\end{aligned}
$$

Here $x$ takes one of the solution $x_{ \pm}$defined below

$$
x_{ \pm}=\frac{-b \pm \sqrt{b^{2}-4 a c}}{2 a}, p_{1}=x \frac{1}{f_{1}-f_{2}}
$$

where $a=1-2 h, b=3 h-1$, and $c=1 /(2 s)-h$. We have for the marginal fitness $f_{1}, f_{2}$ and the fitness $R$

$$
\begin{array}{r}
f_{1}=2 s x+2 \operatorname{sh}(1-x), f_{2}=2 \operatorname{sh} x \\
R=x f_{1}+(1-x) f_{2}=2 s\left[(1-2 h) x^{2}+2 h x\right] .
\end{array}
$$

In terms of the marginal fitness, the original equation (A1) can be written in the form

$$
\begin{aligned}
\frac{d P_{l}}{d t} & =\left(r_{l}-R\right) P_{l}+(L-l+1) P_{l-1} \\
& +(l+1) P_{l+1}-L P_{l}
\end{aligned}
$$

where $P_{l}$ are the class probabilities of the configurations at the Hamming distance $l$ from the reference sequence, $r_{0}=L f_{1}$, and $r_{l}=L f_{2}, l \geq 1$.

Let us denote $x=x_{0}+\delta, R=R_{0}+k_{1} / L$. Then

$$
\begin{aligned}
k_{1} & =2 s[(1-2 h) x+2 h] \delta \\
f_{1} & =2 s x+2 s h(1-x)+2 s(1-h) \delta .
\end{aligned}
$$

For the $p_{1}$, we have the equation

$$
x\left(R(x)+1-f_{1}(x)\right)=\frac{1}{L} p_{1} .
$$


Replacing on the right hand side and before the parenthesis on the left hand side

$$
x \sim\left(1-1 /\left(f_{1}-f_{2}\right)\right)
$$

and

$$
\begin{gathered}
p_{1} \sim\left(f_{1}-f_{2}-1\right) /\left(f_{1}-f_{2}\right)^{2}, \\
\bar{l} \sim 1 /\left(f_{1}-f_{2}-1\right),
\end{gathered}
$$

we find a perturbation expression for $\delta$

$$
\delta=\frac{1}{L\left(R^{\prime}-f_{1}^{\prime}\right)} \frac{1}{f_{1}-f_{2}}+O\left(\frac{1}{L^{2}}\right)
$$

and for $k_{1}=R^{\prime}(x) * \delta$

$$
\begin{aligned}
k_{1} & =\frac{1}{L\left(f_{1}-f_{2}\right)\left(1-\frac{f_{1}^{\prime}}{R^{\prime}}\right)} \\
& =\frac{1}{L\left(f_{1}-f_{2}\right)\left[1-\frac{s-h}{2[(1-2 h) x+h]}\right]} .
\end{aligned}
$$

For $s=5.3, h=0.1, L=4$ we get $R_{n}=8.55$, while $R_{0}+k_{1}=8.49$, thus we have $1 \%$ accuracy.
[1] M. Eigen, Naturwissenschaften 58, 465 (1971).

[2] M. Eigen, J. McCaskill and P. Schuster, Adv. Chem. Phys. 75, 149 (1989).

[3] J. Swetina, and P. Schuster, Biophys. Chem. 16, 329 (1982).

[4] J. F. Crow and M. Kimura, An Introduction to Population Genetics Theory (Harper Row, New York, 1970).

[5] I. Leuthausser, J. Stat. Phys. 48, 343 (1987).

[6] P. Tarazona, Phys. Rev. A 45, 6038 (1992).

[7] N. H. Barton, Evolution 46,551(1992).

[8] T. Wiehe, E. Baake, and P. Schuster, J. Theor. Biol. 177, 1 (1995).

[9] M. Shpak and A. S. Kondrashov, Evolution 53, 600 (1999).

[10] S. Franz and L. Peliti, J. Phys. A 30, 4481 (1997).

[11] E. Baake, M. Baake, and H. Wagner, Phys. Rev. Lett. 78, 559 (1997).

[12] E. Baake and H. Wagner, Genet. Res. 78, 93 (2001).

[13] J. Hermisson, O. Redner, H. Wagner, E. Baake, Theor. Pop. Biol. 62,9 (2002).

[14] D. B. Saakian and C.-K. Hu, Phys. Rev. E 69, 021913 (2004); ibid. 69, 046121 (2004).

[15] D. B. Saakian, C.-K. Hu, and H. Khachatryan, Phys. Rev. E 70,041908(2004).

[16] E. Cohen, D. A. Kessler and H. Levine, Phys. Rev. Lett. 94, 098102 (2005).

[17] D. B. Saakian and C.-K. Hu, Proc. Natl. Acad. Sci. USA 103, 493 (2006).

[18] D. B. Saakian, E. Munoz, C.-K. Hu and M. W. Deem, Phys. Rev. E 73, 041913 (2006).

[19] J.-M. Park and M. W. Deem, J. Stat. Phys. 125, 975
(2006).

[20] J.-M. Park and M. W. Deem, Phys. Rev. Lett. 98, 058101(2007).

[21] K. Sato, K. Kaneko Phys. Rev. E 75:061909(2007).

[22] D. B. Saakian, J. Statis. Phys. 128,781(2007).

[23] D. B. Saakian, Z. Kirakosyan, C.-K. Hu, Phys. Rev. E, 77, 061907 (2008).

[24] E. Munoz, J.-M. Park, and M. W. Deem, Phys.Rev.E 78, 061921 (2008).

[25] Zh. Avetisyan, D.B. Saakian, Phys.Rev.E. 81, 051916 (2010).

[26] R. Burger, The mathematical theory of selection, recombination, and mutation (New York, Wiley, 2000).

[27] W. J. Ewens, Math. Population Genetics (Springer - Verlag, New York, 2004).

[28] G. Bocharov, N. J. Ford, J. Edwards, T. Breinig, S. WainHobson and A. Meyerhans, J. Gen. Virol 86, 3109 (2005).

[29] T. D. Frank,Physica A 331,391(2004).

[30] C.J. Thompson and J.L. McBride, Mathematical Biosciences 21, 127 (1974).

[31] B. L. Jones, R. H. Enns, and R. S. Rangnekar, Bull. Math. Biol. 38, 15 (1975).

[32] The left-hand side of Eq. (13) in [23] should be divided by $N$.

[33] N. N. V. Vijay, Vasantika, R. Ajmani, A. S. Perelson, and N. M. Dixit, J. Gen. Virology 89, 1467,(2008).

[34] C. Escudero and A. Kamenev, Phys.Rev. E 79, 041149 (2009).

[35] M. Assaf and B. Meerson, Phys.Rev. E 81, 021116 (2010). 